\title{
Ueber sulzige Infiltration der Conjunctiva und Sklera.
}

\author{
Von \\ Dr. Walter Schlodtmann, \\ aus Königsberg i. Pr.
}

(Aus der Augenklinik des Herrn Prof. E. Fuchs in Wien.)

Hierzu Tafel III, Fig. 1-4.

Im Laufe der letzten beiden Jahre kamen an der Augenklinik des Herrn Prof. Fuchs drei Fälle einer eigenartigen Erkrankung der Sklera zur Beobachtung, welche in mancher Beziehung an die Skleritis resp. Episkleritis erinnernd, doch so wesentlich von dem typischen Bilde derselben abwichen, dass man es mit einer eigenen Erkrankung zu thun zu haben scheint. $\mathrm{Da}$ in der Literatur nur wenige an den unsrigen erinnernde Fälle vorliegen - ich komme noch auf sie zurïck -, und da ferner eine eingehende mikroskopische Untersuchung eines solchen Falles bisher nicht ausgeführt ist, so will ich versuchen im Folgenden eine Schilderung des Krankheitsbildes sowie eine Darstellung der bei der histologischen Untersuchung gefundenen Resultate zu geben. Leider hatte ich nur den letzten der drei Fälle selbst zu beobachten Gelegenheit, während ich den ersten erst post enucleationem sah, so dass ich nur den. bereits abgeheilten Process am anderen Auge constatiren konnte. 
Herr Prof. Fuchs hatte die grosse Güte, mir den enucleirten rechten Bulbus des ersten Falles zur histologischen Untersuchung zu überlassen; auch stellte er mir die Krankengeschichten der drei Fälle in liebenswürdigster Weise zur Verfügung.

Zur besseren Orientirung schicke ich die wichtigsten Daten aus dem mir vorliegenden reichen Material voraus, ohne mich auf eine detaillirte Wiedergabe der einzelnen Krankengeschichten einzulassen. Nur bei dem ersten, auch anatomisch untersuchten Falle sei eine eingehendere Darstellung des Krankheitsverlaufes gestattet.

\section{Fall I.}

Anamnese: J. W., 74 Jahre alter Pfründner, ist angeblich stets gesund gewesen, bis auf einen bisher dreimal aufgetretenen Rheumatismus der Armmuskeln. Die fünf ersten Kinder des Patienten sind im Alter von $1 / 2-5$ Jahren an unbekannten Krankheiten gestorben, die vier letzten leben und sind gesund. Lues wird negirt. Auch an den Augen ist Patient angeblich früher nie erkrankt. Erst im August 1894 trat spontan eine Entzündung am rechten Auge auf mit zeitweise exacerbirenden Schmerzen. Der Zustand versehlimmerte sich bei Selbstbehandlung mit gelbem Augenwasser. Patient nahm in Folge dessen die Hilfe der Augenklinik in Anspruch und wurde hier ambulatoriseh behandelt, und zwar wegen eines ,skleritischen Knotens“ im inneren Quadranten.

In November 1894 trat eine Infitration am Hornhautrande auf. Aus dieser Zeit datirt der erste Status, welcher hier vollständig wiedergegeben werden mag:

Status praes: R. A. Rings um die Hornhaut, in einer Breite von mehr als $0,5 \mathrm{~cm}$ ist die Bindehaut geröthet, von grossen Gefässen durchzogen und durch eine sulzige rothbraune Verdickung des darunterliegenden Gewebes gleichmässig abgehoben. Einzelne umschriebene Buckel sind nicht vorhanden. Die Conjunctiva seheint ziemlieb innig: mit diesem aus der Episklera hervorgegangenen Gewebe verbunden zu sein, denn sie lässt sich erst weiter rückwärts frei verschieben. Der Limbus schiebt sich von allen Seiten, am wenigsten von aussen unten, auf die durchsichtige Cornea vor. 
Auf den Limbus folgt eine $1 \mathrm{~mm}$ breite Zone, in der die Hornhaut grau getrübt und über das Niveau ihrer übrigen Theile erhaben ist. Die Trübung im Bereiche dieser Zone ist nicht gleichmässig, sondern es finden sich da ganz unregelmässige, saturirte weisse Flecke, zwischen welchen eine geringe diffuse Trübung besteht. Die Trübung scheint die oberflächlichsten Lagen der Hornhaut einzunehmen, grenzt sich gegen die Cornea scharf mit einer etwas buchtigen Linie $a b$ und ist von einzelnen Auslänfern der Gefässe des Randschlingennetzes überzogen. Die übrige Cornea ist gleichmässig matt und leicht trüb, in der unteren Hälfte mit sehr feinen Präcipitaten beschlagen. Die Vorderkammer scheint etwas tiefer als normal, die Pupille wird durch Atropin nur wenig erweitert, lässt jedoch keine deutlichen Synechieen erkenmen und ist rund. Der Fundus ist nicht ganz deutlich zu sehen, zeigt aber keine auffallenden Veränderungen. Temperatur normal. Der Bulbus ist bei Berührung etwas empfindlich.

Patient bekam nun Sublimatinjectionen und, nachdem sich diese als fruchtlos erwiesen hatten, wurden versuchsweise auch Einspritzungen von Jodoformemulsion vorgenommen. Ferner wurde ein Stick der Wucherung am unteren äusseren Hornhautrande excidirt. Davon wurde ein Theil einem Kaninchenauge eingeimpft und reactionslos vertragen. Der Rest wurde geschnitten and zeigte folgendes Bild: Unter einem theils normalen, theils verdünnten Epithelbelag liegen kleinzellige von mono- und polynucleären Leukocyten durehsetzte Wucherungen mit reichlich neugebildeten Lymphgefässen und einzelnen Riesenzellen. An den Stellen, wo die Grundsubstanz wegen geringerer Infiltrirung deutlicher hervortritt, bemerkt man eine feinkörnige Trübung derselben, wie sie auch neuerdings Șchirmer ${ }^{x}$ ) erwähnt, der sie aus der intra vitam bestehenden Durehtränkung des Gewebes mit einer eiweissreichen Flüssigkeit erklärt.

Ende November traten in der nach oben gelegenen braunrothen Masse einzelne ganz kleine verwaschene gelbliche Fleckchen auf, welehe in der Tiefe liegenden gelblichen Knötchen zu entsprechen schienen. Jetzt wurden auch zahlreiche hintere Synechieen constatirt. Um dieselbe Zeit begann auch am linken Ange anscheinend derselbe Process. Es heisst dariber: Im oberen

1) Schirmer, Zur pathologischen Anatomie der Skleritis and Episklexitis. v. Graefe's Arehiv für Ophthalm. 1895, Bd. XLI. 4. S. 160 . 
Theile des Limbus sieht man eine graurothe Verfärbung und ganz leichte Verdickung des Gewebes. Dieselbe reicht nur ganz wenig in die Bindehaut des Bulbus hinein, dagegen über $1 \mathrm{~mm}$ in die durchsichtige Hornhaut; sie endet mit einer grauen Zone, in welche saturirte grane Punkte gelagert sind. Im Uebrigen ist das Auge normal.

Ende December beginnen rechts auch in den durchsichtigen Theilen der Hornhaut zarte, oberfiächliche Trübungen aufzutreten, das Epithel über der ganzen Cornea ist matt und uneben. Am linken Auge ist die Wucherung am Limbus merklich breiter geworden, bis zu $1^{3 / 4} \mathrm{imm}$. Das sulzige Gewebe liegt ganz der Hornhaut anf; die auf der Sklera liegende Bindehaut zeigt vielleicht nur unmittelbar am Rande der Hornhaut eine übrigèns nicht einmal mit Sicherheit festzustellende Infiltration.

Da dureh die Behandlung mit Sublimat- und Jodoforminjectionen keine wesentliche Besserung zu erzielen war, bekam Patient seit Februar 95 Jodkalium. Rechts ist die Infiltration jetzt recht breit und reicht, namentlich nach oben innen, weit hinauf, so dass der ganze obere innere Quadrant der Conj. bulbi wie von einem ganz flachen skleritischen Knoten eingenommen aussieht. Die Farbe der sulzigen Infiltration ist dunkelbraunviolett, die Sehwellung oben innen etwas empfindlich. Die gelblich-weissen Infiltrate liegen nicht mehr ganz regelmässig dem Rande entlang, sondern gruppenweise, so dass ein Theil derselben, vom Rande entfernt, mehr schon in der sulzigen Masse liegt. Diese zeigt überdies näher dem Hornhantrande kleine oberfläehliche spiegelnde Stellen, die vielleicht als kleine aus dem Zerfall von Infiltraten hervorgangene Substanzverluste aufzufassen sind. Patient hat tiefe, in die Stirn ausstrahlende Schmerzen, die bisweilen ziemlich heftig sind. Links hat sich die sulzige Auflagerung auf dem obersten Theil ganz abgeflacht, ist fast gefässlos, und die kleinen randständigen Infiltrate sind spurlos verschwonden.

Etwa dasselbe Bild bot Patient auch noch, als er im Mai 95 in die Klinik aufgenommen wurde: Sein Visus war damals:

R.: Finger zählen in $1 \mathrm{~m}$;

L.: $6 / 36 ;+1,5 \mathrm{D} 6 / 6 ;+4,5 \mathrm{D}$ Sn 0,5 .

Die von Herrn Prof. Ludwig ausgeführte Harnanalyse ergab eine starke Vermehrung von Indican; abnorme Stoffe fehlten bis auf minimale, quantitativ nicht nachweisbare Spuren Zucker.

Am 19. V. wurde wegen der nun fast vollständigen An- 
wachsung des Pupillarrandes an die Kapsel rechts eine Iridektomie gemacht.

Auch bei der Entlassung, Mitte Juni, war der äusserliehe Befund unverändert. Visus rechts: Lichtempfindung in $2,5 \mathrm{~m}$, nach innen nicht vorhanden.

Schon nach ein paar Tagen suchte Patient wegen Schmerzen im rechten Auge die Klinik von Nenem anf, ohne sonstige Veränderungen im Befunde. Es wurde in Folge dessen rechts die Enucleation vorgenommen, und Patient nach fieberfreiem Wundverlauf entlassen.

Als ich Patient im Januar 96 zum ersten Male sah, gab er an, dass er vor etwa zwei Monaten im linken Auge leichte Schmerzen gehabt habe, die aber nach einigen Tagen ohne Behandlung spontan wieder vergangen seien. Das linke Auge bot damals folgendes Bild: Bulbus völlig reizfrei. Die Sklera ist im Umkreise der Hornhaut im Grossen and Ganzen weiss, nur an einer etwa linsengrossen ca. $4 \mathrm{~mm}$ vom inneren Hornhantrande entfernten Stelle erscheint sie bläulich durchscheinend und lässt daselbst mehrere besonders dunkle verticale Streifen erkennen. Nach oben und unten setzt sich diese bläuliche Stelle in Form eines dem Limbus parallel verlaufenden $1-2 \mathrm{~mm}$ breiten Streifens von gleicher Färbung fort. Eine ähnliche Stelle findet sich in der Nähe des äusseren Hornhautrandes. Eine Niveauerhebung der Sklera ist nirgends vorlanden. Einige in der Conjunetiva verlaufende Gefässe, besonders in der oberen Bulbushälfte, senken sich ausserordentlich nahe dem Limbus in die Sklera, eines derselben sogar in den limbus selbst. Dieser letztere ist deutlich zugeschärft und greift in Form einer leichten Trübung auf die Hornhaut über. Die Trübung ist oben in der Mitte des Hornhautrandes am breitesten, ea. $2 \mathrm{~mm}$, und geht, sich nach beiden Seiten verschmälernd, als weissliehe, ziemlich scharf begrenzte Linie in den normalen Limbus über. Die Trübung zeigt bei Betrachtung mit unbewaffnetem Auge eine deutlich radiäre Streifung, welche sich bei der Untersuchung mit einer starken Loupe in zahlreiche faine, oberfläehliche Gefässe auflösen lässt. An die in der ätrsseren Limbushälfte befindliche linienförmige weisse Trübung setzt sich nach innen $z \mathfrak{u}$, von ihr dureh eine schmale durehsichtige Zone getrennt, eine ungefähr sichelförmige zarte, oberflächliche Trübung mit undentlichem Contour an. Sonst erscheint das Auge normal; der Fundus bietet keine pathologisehen Veränderungen.

$$
\mathrm{S}^{6} / 36 ;+2,0 \mathrm{D}^{6} / 8 ;+5.0 \mathrm{D} \text { Jaeg. Nr. II. }
$$


Fall II.

Anamnese: J. H., 64 Jahre alter Tagelöhner, erkrankte angeblich vor zwei Jahren im Anschluss an eine Influenza an Rheumatismus mit Selmerzen und pelzigem Gefühle in den unteren Extremitäten. $\mathrm{Zu}$ gleicher Zeit stellte sich eine heftige Entzündung beider Augen ein, welche mit starken Schmerzen und Herabsetzung des Sehvermögens einherging. Die auch jetzt noch bestehenden Schmerzen beschrïnken sich auf Augen und Stirn und treten anfallsweise mit Intervallen von einigen Tagen auf. Seit fünf Wochen hat sich das Sehvermögen auffallend verschlechtert. Lues wird negirt.

Status: Im Harn weder Zucker noch Eiweiss. R. A. Die Conjunetiva ist in ihrer ganzen Ausdehnung stark injicirt und sondert ein glasig-schleimiges Secret ab. Im Ganzen Umkreise der Hornhaut besteht eine sulzige Infiltration der Conjunctiva und des episkleralen Gewebes, in Folge dessen die Bindehaut ganz unbeweglich an die Unterlage angeheftet ist. Die Breite dieser Zone ist nicht überall gleich; im Allgemeinen ist sie nach oben und unten breiter als nach den Seiten. Die durehschnittliche Breite beträgt $6-7 \mathrm{~mm}$. Ihr peripherer Rand ist nicht ganz scharf, wird aber noch dadurch besser markirt, dass ausser der Farbenveränderung auch eine Niveaudifferenz besteht. Es sieht nämlich so aus, als ob die infiltrirte Parthie gegenüber der normalen Sklera eingesunken sei. Die centrale Grenze dieser Zone ist auf die Hornhaut hinaufgerückt, und zwar oben am meisten, ea. $2 \mathrm{~mm}$ oder mehr. Die Infiltration sehärft sich hier zu und endigt mit einem scharfen, buchtigen Rande. Das durchsichtige Areal wird dadurch eingeengt und bildet ein unregelmässiges liegendes Oval. Innerhalb der infiltrirten Zone sieht die Bindehant gleichmässig grauroth aus; mit der Loupe sieht man zahlreiche feinste Gefässe, welche überall aus der Tiefe zu der Oberfläche emporkommen. Die zuführenden episkleralen Gefässe sind sehr stark exweitert. Der Druck der Knopfsonde hinterlässt für einige Zeit ein kleines Grübchen. Die Cornea ist, soweit sie nicht von Infiltrationen überzogen ist, nur ganz leicht getrübt. Die Iris, bis an die Hornhant vorgetrieben, atrophisch, von oberflächlichen Gefässen durchzogen, zieht sich gegen die Pupille trichterförmig: nach rückwärts; die Pupille ist durch eine feine graue Membran verschlossen, in der auch Gefässe zu sehen sind. Die Linse scheint ebenfalls getrübt zu sein. 
L. A. bietet im Grossen und Ganzen dasselbe Bild. T. beiderseits -2 . Visus:

R.: Lichtempfindung in $5 \mathrm{~m}$; Projection richtig.

L.: Lichtempfindung in $40 \mathrm{~cm}$; Projection nur nach aussen.

6. VI. Links wird ein Stückchen der Conj. bulbi excidirt, rechts eine Iridektomie gemacht. Bei der nach 14 Tagen erfolgenden Entlassung bietet Patient dasselbe Bild; nur ist reehts Lichtempfindung bloss noeh in $1 \mathrm{~m}$ Entfernung vorhanden.

\section{Fall III.}

Anamnese: A. S., 74 Jahre alter Privatmann, wurde vor sieben Jalren an einer hiesigen Augenklinik links wegen Cataracta senilis extrahirt; vor sechs Jahren wurde er dann auch rechts, und zwar in Jassy (Rumänien), operirt. 14 Tage nach dieser zweiten Operation sah er noch, dann bekam er Schmerzen in dem betreffenden Auge, welche zehn Tage dauerten. Er wurde mit Augenwasser, Umschlägen, Stirnsalbe und Blutegeln hinter dem Ohr behandelt. Die Schmerzen hörten auf, das Sehvermögen war jedoch fast vollständig vernichtet. Seit neun Monaten hat er wieder drǘckende Schmerzen im rechten Auge, welche nach der Sehläfe, den oberen Zälnen und der Nase hin ausstrahlen und bei eintägiger Dauer in zweiwöchentlichen Intervallen auftreten. In den freien Intervallen besteht geringer Stirnkopfschmerz.

Status: Das rechte Auge zeigt zunächst mannigfache trachomatöse Veränderungen, die hier nicht interessiren. Die Conj. bulbi ist im Umkreise der Cornea durchweg von eigenthümlich schmutzig-rother Farbe nnd sulzig, durchscheinender Beschaffenheit. Sie ist von zablreichen theils feinen, theils stark erweiterten und geschlängelten Gefässen durchzogen. Die Breite der so veränderten Zone ist oben $8 \mathrm{~mm}$, aussen $5 \mathrm{~mm}$ (hier ist die Abgrenzung gegen die Peripherie eine verschwommene); unten reicht die Zone bis zur Uebergangsfalte und innen fast bis zur Carunkel. Am intensivsten ist die Veränderung innen und unten ausgebildet. Die Gefässe der tieferen Schichten scheinen blasser durch. Eine ähnliche graue sulzige Infiltration sehiebt sich von allen Seiten auf die durchsichtige Cornea, am weitesten innen, etwa $3 \mathrm{~mm}$. Im Algemeinen grenzt sieh die Trübung gegen die durchsichtige Hornhaut durch eine vielfach gebuchtete Linie $a b$, indem die Infiltration mit einer $0,5 \mathrm{~mm}$ breiten ziemlich zusammenhängenden grauweissen Randzone endigt. Nur oben finden sich nahe der beschriebenen Zone 
schon im Bereich der durchsichtigen Cornea mehrere kleine granweisse, oberflächliche Trübungen. $\mathrm{Zu}$ diesen Trübungen ziehen zahlreiche dendritisch verzweigte Gefässe, welche die Randzone nicht zu überschreiten scheinen. Mit der Loupe jedoch sieht man, dass, besonders aussen unten, viele Gefässe die Randzone überschreiten, um noch eine Strecke weit in die Hornhaut zu ziehen, welche an dieser Stelle keineswegs vollkommen durchsichtig; sondern von feinsten, dichtstehenden, oberfläehlichen grauen Fleckchen durebsetzt ist. Innen ist diese Abgrenzung gegen die durchsichtige Hornhaut weniger scharf.

Kammer tief. Iris grünlich verfärbt, mit wenig deutlicher Zeichnung. Colobom nach oben. In Pupille und Colobom befindet sich eine weisse Membran; im Bereich des Coloboms ist sie mehr bläulichweiss und grenzt sich gegen die Pupille zackig $a b$, in der Pupille erscheint sie als zartgrane Membran. Daselbst ein sehr breiter, unregelmässiger Pigmentsaum. T. - 3 . Visus: Handbewegungen; Lichtempfindung in $5 \mathrm{~m}$ gut; Projection unsicher.

Das linke Auge bietet, abgesehen von den trachomatösen Veränderungen und einer Cataracta secundaria, nichts Besonderes. Fundus normal. Visus:

$+4,5 \mathrm{D}$ cyl. A. hor. $\bigcirc 9,0 \mathrm{D}$ sph. $6 / 1 \mathrm{~g}$.

$+4,5$ D cyl. A. hor. $\bigcirc$ 13,0 D sph. Jaeg. No. III.

10. I. Iridektomie nach oben am rechten Auge.

18. I. Entlassung. Rechts ist die sulzige Infiltration der Conj. bulbi etwas geringer, die Wucherung am Limbus nicht vorgerückt. Schwarz erscheinender klaffender Spalt durch die Cataractmembran. Visus: sph. ${ }^{6} / 30$.

R.: Fingerzählen in $0,5 \mathrm{~m} ; 4,0 \mathrm{D}$ cyl. A. hor. $\bigcirc 12,0 \mathrm{D}$

L.: Idem, wie vorher.

Wenn wir aus dem Krankheitsbilde, wie es sich aus diesen drei Fällen mit grosser Uebereinstimmung ergiebt, noch einmal die wesentlichsten Punkte, durch welche die beschriebene Erkrankung ausgezeichnet ist, kurz zusammenfassen, so ist das Charakteristische, von der typischen Form der Skleritis resp. Episkleritis Abweichende einmal jene sulzige, succulente Beschaffenheit des infiltrirten Hornhautrandes, welche sehr auffallend ist und dem 
Auge ein ganz eigenartiges Gepräge verleiht. Ferner ist die Localisation und Ausdehnung des Infiltrates entschieden ungewöhnlich. Denn während es wohl bei der tiefen Form des Skleritis hier und da vorkommt, dass die Entzündung die ganze Hornhaut ringförmig umschliesst, so ist das doch schon etwas Ungewöhnliches, und sie liegt dann auch - zum Unterschied von lymphatischen Knoteu - niemals im Limbus selbst, sondern lässt ihn frei. Hier dagegen ist der Hornhautrand recht eigentlich der Sitz des Krankheitsprocesses, und von ihm greift die Infiltration nach beiden Seiten a uf die benachbarten Membranen über, indem sie nach der Hornhaut zu, dieselbe zum Theil überlagernd, sich mit scharfem Rande abgrenzt, nach der Sklera hin dagegen sich allmählich abflacht und unmerklich in das normale Gewebe übergeht. Charakteristisch ist endlich im weiteren Verlauf der Krankheit das allmähliche Weiterschreiten der Infiltration im Hornhautgew ebe, so dass das durchsichtige Gebiet der Cornea dadurch mit der Zeit auf ein immer kleineres Areal reducirt wird. Auch ist der einmal vorhandene entzündliche Zustand der Corneoskleralgrenze viel beständiger als die Entzündung der Sklera bei typischer Skleritis and lässt sich durch die Therapie viel weniger beeinflussen, sodass es höchstens gelingt, denselben am Weiterschreiten zu verhindern und auf dem Status quo zu erhalten, während eine wesentliche Besserung oder gar Heilung, nur in den allerfrühesten Anfangsstadien möglich zu sein scheint (vgl. Fall I, linkes Auge).

Sehen wir uns in der Litteratur nach Fällen um, wo eine ähnliche Infiltration des Limbus beschrieben wird, so finden wir nur sehr wenige Analoga. Und wenn dieselben auch in einzelnen Punkten eine bemerkenswerthe Uebereinstimmung mit dem von uns beobachteten Bilde aufweisen, so ist doch keines unter ihnen, welches ätiologisch dem Unsrigen an die Seite gestellt werden könnte. 
So beschreibt Donald Gunn ${ }^{1}$ ) drei Fälle von wahrscheinlich syphilitischer Infiltration des Limbus. Besonders beim zweiten Falle, erinnert die Schilderung, welche er von der Conjunctiva macht, an unsere Fälle. Er sagt von ihr: $"$... its surface presenting a smooth waxy appearence of the colour of pink coral. The swollen conjunctiva was raised slightly above the level of the corneal margin, which it slightly overlapped." Abweichend ist also nur die hellere Farbe; auch betrifft die Veränderung augenscheinlich nur die Conjunctiva und Subconjunctiva, da sie auf der Sklera frei verschieblich ist. Bei allen drei Patienten war Lues nachzuweisen.

Eine noch grössere Uebereinstimmung zeigt der von. Brailey ${ }^{2}$ ) veröffentlichte Fall, wo es sich um ein Kind mit subacuter Lymphadenitis handelt. Die Infiltration des Limbus, welche dabei besteht, bezeichnet Brailey als "a flattened, band-like thickening of the ocular conjunctiva" und sagt ron ihrer Ausdehnung: "The thickening gradually dimishes both towards the corneal edge, where it ends abruptly $5 \mathrm{~mm}$ above the corneal level, and peripherally, in which direction it shelves gently away". In diesem Fall hatte die Infiltration auch die von uns beobachtete charakteristische schmutzigröthliche Färbung und war mit der Sklera fest verwachsen. Ganz interessant ist dann der spätere Verlauf: es bilden sich Geschwüre, welchen correspondirende Ulcerationen an der Innenfläche des Lides entsprechen, so dass es an dieser Stelle zu Adhäsionen zwischen Bulbus und Lid kommt; die Infiltration zeigt bald

1) Donald Gunn, Transactions of the opthalmological society of the united kingdom. 1894. Vol. XIV: 1. Syphilitic (?) subconjunetival infiltration. pag. 68. 2. Syphilitic (?) infiltration of conjunctiva. p. 68-71. 3. Syphilitic (?) infiltration of ocular conjunctiva. p. $71-72$.

9) W. A. Brailey, Peculiar solid thickening of the ocular conjunctiva in a child apparently tubercular. Transactions 1889. Vol. IX, p. $82-85$.

พ. Graefe's Archiv für Ophthalmologie. XuIII. 1 . 
eine graue Verfärbung, und es treten unregelmässige Knötchen darin auf. Ebenso ist das histologische Bild ein anderes, welches „mit der Annahme der tuberculösen Natur übereinstimmt", nämlich Riesenzellen und käsige Degeneration erkennen lässt, ohne dass freilich auch dort der Nachweis von Tuberkelbacillen gelungen wäre.

Eine gänzlich abweichende Aetiologie haben die Fälle der französischen Autoren, Coppez ${ }^{1}$ ) und Gayet ${ }^{2}$ ). Da hier die Verdickungen nicht durch reine Infiltrationen, sondern durch Tumoren resp. Tumorenmetastasen bedingt sind, so gehören die Fälle eigentlich nicht direct hierher. Ich muss sie aber dennoch besprechen, weil sie äusserlich ausserordentlich an unser Krankheitsbild exinnern. Man höre die Beschreibung Coppez's von dem Aussehen des Limbus: "Le limbe cornéen a disparu. Il n'y a aucune limite précise entre la sclérotique ef la cornée, mais il existe à cheval sur les deux membranes une substance blanc rosée qui se prolonge sous la conjonctive". Und ähnlich äussert sich Gayet: „Sur le limbe de la cornée existait un cercle d'infiltrats arrondis de couleur blanche ou blanc jaunâtre, plus ou moins saturée, se touchant presque tous et formant anneau autour d'une partie centrale parfaitement nette et transparente".

Gayet führt dann noch zwei seinen beschriebenen analoge Fälle auf, bei denen es sich seiner Ansicht nach um denselben Process handelt, die jedoch zu kurz skizzirt sind, als dass man sich eine klare Vorstellung von ihnen machen könnte. Jedenfalls ersieht man aus den besprochenen Fällen, dass die Symptome, wie sie auch unsere Patienten dar-

1) Coppez, Tumour orbitaire avec envahissement secondaire de la sclérotique et de la cornée. Archives d'ophthalmologie 1895, pag. $544-565$.

2) Gayet, Denx tumeurs symétriques des globes oculaires. Archives d'ophthalm. 1888, pag. 18-28. 
bieten, keineswegs der Ausdruck einer bestimmten Erkrankung sind, sondern dass ihnen die verschiedenartigsten ätiologischen Momente zu Grunde liegen können. Wenn wir daher auch, wie ich später zu zeigen gedenke, die aus der Titeratur sich ergebenden ätiologischen Ursachen, wie Syphilis, Tuberculose und auf der Basis gewisser Constitutionsanomalieen (Pseudoleukämie?) sich entwickelnde Tumoren, bei unserem ersteren, histologisch untersuchten Falle wohl mit Sicherheit ausschliessen können, so bleibt doch immerhin die Frage offen, ob alle drei beschriebenen Fälle als eine und dieselbe Krankheit aufzufassen sind, und ob nicht vielleicht die beiden letzten ätiologisch in eine der oben erwähnten oder in eine noch andere, neue Kategorie gehören; eine Frage, welche natürlich nur an der Hand einer genauen mikroskopischen Untersuchung zu entscheiden ist.

Verlassen wir das klinische Bild und wenden wir uns dem anatomischen Befunde zu, wie er sich uns im ersten Falle darbietet, so ergiebt sich hier keine so auffallende Uebereinstimmung mit den aus der Literatur gesammelten Fällen, wie dort; und wo eine solche vorhanden ist, bezieht sie sich nur auf nebensächlichere Veränderungen. Im Grossen und Ganzen weichen die anatomischen Bilder, wie sie bisher beschrieben sind, im Gegentheil sehr stark von unserem Befunde $a b$, soweit ich das wenigstens nach einem Coppez'schen Präparate, das ich durch die Güte des Herrn Prof. Fuchs selbst zu besichtigen Gelegenheit hatte, beurtheilen kann. Näherliegend und zahlreicher sind die Beziehungen, welche zu den bisher veröffentlichten Fällen von histologisch untersuchter Skleritis und Episkleritis bestehen, welche wenigstens in Finzelheiten an unsere Resultate erinnern, wenn auch das Gesammtbild keine rechte Uebereinstimmung zeigt.

Was nun dieses anatomische Bild betrifft, so war äusserlich an dem enucleirten, in Formol und Alkohol gehärteten Bulbus, abgesehen von einem artificiellen Loch in der Cornea in 
Folge einer Trepanation ${ }^{1}$, nichts Besonderes wahrzunehmen. Durch einen horizontalen Sehnitt unterhalb der Sehnerven halbirt, bot er auf dem Durchschnitte folgendes Bild (vgl. auch Fig. 1 und 4): Die Linse ist in die vordere Kammer luxirt (Fig. 1a) und zeigt einen mässig grossen, etwas dunkler gefärbten Kern. An der vorderen Fläche befinden sich zwei kerbenförmige Defeete. Sowohl jene wie diese Veränderung sind augenscheinlieh ebenfalls auf die Trepanation zuriickzuführen. Die nach hinten zurïckgedrängte Iris (Fig. 1b) liegt der hinteren Linsenfäche an. Die Chorioidea und Retina sind rechts und links in ausgedehntem Maasse durch gelbliche Exsudatmassen von der Sklera abgehoben und zum Theil fast bis in das Centrum des Glaskörpers gedrängt (Fig. $1, d \mathbf{n} . c)$. Auch eine Netzhautablösung in eigentlichem Sinne besteht an verschiedenen Stellen, indem hier und da die Netzhaut von der schon abgehobenen Chorioidea ihrerseits dureh Exsudatschichten getrennt ist. Der Glaskörper ist stark geschrumpft und zu zahlreichen bald dünneren, bald dickeren Membranen oder compacteren Massen verdichtet, welche fast durchweg eine rein weisse Farbe zeigen.

Der in Celloidin gebettete Bulbus wurde im Bereich der Cornea in horizontale Serienschnitte zerlegt, und die Sehnitte zum grössten Theil mit Hämalaun und Eosin gefärbt. Daneben wurden Parallelfärbungen vorgenommen: 1. mit Alaunearmin, 2. Hämalaun und Fuchsin-Pikrinsäure nach van Gieson, 3. mit Indigocarmin nach Norris-Shakespeare und 4. auf Markscheiden nach Weigert. Bakterienfärbungen wurden nach Löffler, Weigert, und auf Tuberkelbacillen ansgeführt, und zwar ergaben sie alle negative Resultate.

Der mikroskopische Befund war folgender:

Die Cornea ist - abgesehen von jenen Sehnitten, an welchen der durch die Trepanation hervorgerufene Defect vorhanden ist - in der nasalen Hälfte zum grössten Theil mit Epithel bekleidet; gegen die Mitte löst sich letzteres dann los und liegt als kurzer schmaler Streifen frei. Auf dieser Seite ist anch die Grenze zwisehen Corneal- und Conjunetivalepithel deutlich erkennbar. Auf der temporalen Seite ist das Epithel (vielleicht artifciell in Folge der Trepanation) anf eine Strecke hin

1) Da die Hornhaut in ihren centralen Parthieen noch ganz normal und transparent erschien, so wurde dieselbe unmittelbar im Anschluss an die Enucleation trepanirt, und das so erhaltene Stück für einen Fall ron Leukom benutzt. 
nicht vorhanden, erst gegen den Limbus zu findet es sich wieder, doch lässt es sich hier nicht mehr scharf gegen das Conjunctivalepithel abgrenzen. Im ganzen Umkreis um die Hornhaut ist der Epithelbelag in der Nähe des Limbus nieht unerheblich verdickt und senkt sich vielfach in Form solider oder trichterförmiger Zapfen in die Tiefe (Fig. 4a), indem er die Lamellen des Hornhautparenchyms leicht auseinanderdrängt und sich zwischen ihnen zungenförmig weiter vorschiebt. Etwa durch den Schnitt getroffene Lumina solcher Epitheltrichter sind zum Theil mit Zerfallsproducten, Schleim, Pigmentkörnchen, abgestossenen Epithelien ete. erfüllt. Die diese Epithelzapfen umgebende Matrix weist regelmässig einen bald sehmäleren, bald breiteren Streifen infiltrirten Gewebes auf.

Ein wesentlich anderes Bild bietet eine in der Nähe des Hornhauteentrums befindliche Epitheleinsenkung. Hier ist ein Substanzverlust im Hornhautgewebe vorhanden der mit Epithelzellen überkleidet und ausgefüllt ist. Und wem sich anch hier in ähnlicher Weise eine Epithelwucherung zungenförmig in dem Hornhautparenchym centralwärts vorsehiebt, so ist doch überall eine Continuitätsunterbrechung sowie eine regellose Lagerung der Cornealamellen $\mathrm{zn}$ constatiren, während letztere durch jene Epithelzapfen nur sanft anseinandergedrängt und in ihrem Verlauf nicht unterbrochen sind. In dem diese Epitheleinsenkung umgebenden leicht infiltrirten Gewebe, sowie auch an anderen oberflächlicher gelegenen infiltrirten Stellen der Hornhaut liegen einige typische grosse Riesenzellen, daneben eine Anzahl kleinerer Gebilde, welche die Anfangsstadien von Riesenzellen darzustellen scheinen.

In der ganzen unteren Hälfte der Hornhaut findet sich eine Pigmentanhäufung im Epithel. Und zwar beschränkt sich dieselbe nicht in physiologiseher Weise auf die unmittelbare Nähe des Limbus, sondern erstreekt sich auch weiter auf die centraleren Parthieen. Das Pigment liegt in Form feinkörniger Conglomerate in dem Protoplasma der Zellen, meistens dicht neben den Zellkern gelagert, und ist besonders reichlich in den Basalzellen vorhanden, so dass es hier einen förmlichen Basalsaum bildet. Vereinzelt findet es sich als isolirte Haufen auch im oberfläehlichen Hornhautparenehym.

Die Bowman'sche Membran lässt sich nach innen zu bis zu ihrem normalen Rande verfolgen, nach aussen hört sie schon viel friiher auf. Von dem sie bekleidenden Epithelbelag ist sie fast in ihrer ganzen Ausdehnang durch eine zellreiche, 
grösstentheils längliche, doch auch runde Kerne führende bindegewebige Schichte getrennt, die nach dem Rande der Bowman'schen Membran zu an Mächtigkeit zunimmt und sich continuirlich in das infiltrirte subconjunctivale Gewebe fortsetzt. Gefässe waren nur in der äussersten Peripherie in selur spärlicher Anzahl und von geringem Kaliber zu constatiren. Mehr centralwärts verjüngt sich diese Zwischenlagerung, fehlt jedoch vollständig nur. auf kurze Strecken, da vielfach anch an den Stellen, wo das Epithel der Bowman'schen Membran direct aufzuliegen scheint, sich hier und $\mathrm{da}$ in unregelmässigen Zwischenräumen noch intensiv gefärbte Rundzellen einzeln oder in Gruppen zu zweì bis drei nnter den basalen Epithelzellen vorfinden.

In der Nähe des inneren Randes ist dieser Pannus an einer Stelle besonders kernreich und verbreitert sich $\mathrm{zu}$ einem Infiltrate, das die Bowman'sche Membran durchbricht und sich auch noeh in den oberflächlicheren Hornhautlamellen etablirt (Fig. 4b). Auch die Epitheldecke wird von ihm in Mitleidenschaft gezogen, indem sich die neugebildete infiltritte Bindegewebsschichte in melhreren (bis zu drei) übereinanderliegenden dünnen. Platten dexart zwischen die Epithelzellen schiebt, dass sie dieselben nicht nur von der Bowman'schen Membran abhebt, sondern sie auch noch in mehrere isolirte Lagen trennt. An anderen Stellen, ebenfalls in der Nahe der Corneoskleralgrenze, schiebt der Pannus kleine bindegewebige Zapfen zwischen die Epithelzellen.

Das Hornhautparenchym ist in seinen centralen Parthieen ziemlich normal; es zeigt im Grossen und Ganzen eine regelmässige Anordnung seiner Fasern, und nur hier und da sieht man vereinzelte Leukocyten. Erst nach dem Rande zu finden sich zusammenhängendere, entweder sich zwischen Hornhantlamellen hinziehende streifenförmige oder um die Gefässe gruppirte eireumscripte Infiltrate, welche dann peripherwärts in die Infiltration der Sklera übergehen. Diese Infiltrate sind in der äusseren Hornhauthälfte viel zablreicher und erstrecken sich auch weiter centralwärts als auf der inneren Hälfte. In den hintersten Corneaschichten findet sich, unmittelbar in der Nähe des Kammerwinkels, also in seiner vorderen Wand, ein die ganze Hornhantmitte umgebendes ringförmiger Infiltrat (Fig. $4 e$ ), das an einzelnen Stellen etwas an Mächtigkeit abnimmt, jedoch überall, auch in den vertieal geführten Schnitten, vorhanden ist. An einzelnen Schnitten lässt sich zwischen diesem und dem oberflächlichen Infiltrat des Limbus eine Verbindung längs eines Gefüsses verfolgen. 
Ueber sulzige Infiltration der Conjunctiva und Sklera.

Die Descemetische Membran ist in der ganzen Ausdehnung der Hornhaut vorhanden and bietet nichts Besonderes.

Das ihr aufliegende Endothel erscheint etwas flach, scheint aber völlig normal zu sein. Nur an einzelnen Stellen sind ihm kleine Exsudatmassen aufgelagert. Erst an dem Kammerwinkel findet sich eine ausgedehntere Auflagerung von Rundzellen und Pigmentkörnchen auf dem Endothel, sowie eine Zwischenlagerung derselben Elemente zwischen diesem und der Descemetischen Membran.

Das Conjunctivalepithel ist ein wenig verdickt, sonst aber normal und zieht in gleichmässiger Lage über die erkrankten Parthieen der Episklera und Sklera hinweg. Die pathologisehen Veränderungen der letzteren sind folgende:

Im ganzen Umkreis um die Hornhaut breitet sich ringförmig eine Infiltration aus, welche, unmittelbar hinter der Corneoskleralgrenze liegend, die Episklera und oberflächlichen Skleralschichten einerseits (Fig. 4, d) und das Corpus eiliare, Suprachorioidea und tiefste Skleralamellen andererseits (Fig. 4,e) betrifft. Bei der Schilderung der Ausbreitung dieser Infiltrationen und ihrer Lagerung zu den Geweben halte ich mich im Wesentlichen an das Bild, wie es silch in den centralgelegenen Horizontalschichten präsentirt. Hier weisen dieselben in ihrer Localisation and Beschaffenheit auf beiden Seiten ziemlich bedeutende Verschiedenheiten auf.

In der inneren Hälfte hat die Entzündung in den oberflächlicheren Regionen ein zusammenhängendes massiges Infiltrat gesetzt, dessen oberste Lagen nach vorn bis zum inneren Rand der Bowman'schen Membran reichen, wo sie zum Theil in den Pannus übergehen, zum Theil sich ziemlich seharf gegen das normale Hornhautparenchym absetzen, und sich nach hinten bis zur Ansatzstelle der Muskelsehne erstrecken. Im Ganzen beträgt die Ausdehnung der Infiltration von vorn nach hinten hier etwa $8 \mathrm{~mm}$. In dem Grade, wie die Infiltration in die Tiefe steigt, nimmt sie auch an Zellreichthum $\mathrm{zu}$, so dass sie an ihrer breitesten Stelle, oberhalb des Corpus ciliare, wo sie über die Hälfte der Sklera einnimmt, auch am zellreichsten ist und hier das Grundgewebe vollständig verdeckt. Ziemlich direct unter dem Conjunctivalepithel sind die Lymphgefässe auffallend vermehrt und zeigen stellenweise eine en orme Ektasie (Fig. $4, f$ ). Ebenso hat eine Vermehrung und Ektasie der Blutgefässe stattgefunden, doch liegen dieselben nicht so oberflächlich, wie die Lymphgefässe, sondern mehr in den tieferen Schichten der 
Infiltration. Die überall mit Endothel ausgekleideten Wände der Blutgefässe scheinen normal zu sein, wenigstens ist es nicht möglich, Wucherungen, hyaline Degenerationen oder atheromatöse Veränderungen an irgend einer Stelle nachzuweisen. Doch lässt sich an vielen Stellen constatiren, dass die Infiltration längs der Gefässe weiterkriecht. $\mathrm{Da}$, wo die Infiltration besonders weit in die Tiefe vorgedrungen ist, finden sich, unmittelbar neben den normalen Sklerabündeln liegend, schön ausgebildete grosse Riesenzellen.

Von der normalen Sklera (Fig. $4, g$ ) sind an dieser Stelle nur wenige Lamellen erhalten, welche die obere von der tiefen Infiltration trennen. Doch besteht ein Zusammenhang zwischen beiden durch mehrere die Gefässe begleitende Infiltrationsstränge (Fig. 4, h).

Die tiefer gelegene Infiltration hat ihren Hauptsitz im Corpus eiliare, Suprachorioidea und inneren Skleralamellen und lässt diese Gebilde stark angesehwollen erscheinen. Doch erstreckt sie sich auch in die Iris hinein, und da sie mit dem oben erwähnten an dem hinteren Hornhautrande liegenden Infiltrate in Verbindung steht, so ist die ganze Kammerbucht von infiltrirtem Gewebe begrenzt. Im Uebrigen ist letztere frei und eine Verwachsung oder Anlagerung der Iriswurzel an die Cornea nirgends nachweishar. Der Fontana'sche Raum zeigt keinen so colossalen Zellreichthum, wie z. B. der Ciliarkörper, dessen Structur stellenweise vollständig durch die Rundzellen verdeckt ist, sondern lässt überall die welligen Bindegewebsbündel des Ligamentum pectinatum deutlich erkennen. Die Ciliarfortsätze zeigen vielfach sklerorotische und in Folge dessen von der Infiltration ziemlich verschont gebliebene Stellen, dagegen finden sich andererseits auch wieder circumscripte, ziemlich dichte kleine Zellenhäufungen.

Inmitten der im Corpus ciliare und der Suprachorioidea liegenden Infiltration fallen mehrere, zum Theil recht ausgedehnte Stellen auf, welche sich intensiv mit Hämalaun gefärbt haben und ein eigenthümlich fleckiges Aussehen besitzen (Fig. 4, i). Die Untersuchung mit stärkerer Vergrösserung ergiebt, dass diese Stellen aus Ansammlungen von fragmentirten, unregelmässig gestalteten and $z u$ unregelmässigen Körnern und Klumpen gruppirten Kernen besteht. Das Grundgewebe ist daselbst getrïbt, opak und nur wenig durchscheinend, so dass die ursprüngliche Structur nicht mehr dentlich $\mathrm{zu}$ unterseheiden ist. Vielfach lagern sich an die Peripherie solcher Stellen, welche zweifellos als Gewebsnekrose aufzufassen sind, Riesenzellen bald in grösserer, 
bald in geringerer Menge an. Fig. 3 zeigt eine solehe Stelle mit besonders zahlreichen and schön entwickelten Riesenzellen.

Die Zellen der Infiltrationen sind vorwiegend mononucleäre Leukocyten, doch finden sich auch nicht unerhebliche Mengen polynucleärer. Und zwar scheinen letztere mit Vorliebe die Gefässe zu begleiten, auf deren unmittelbare Nähe sie sich beschränken, so dass sie stellenweise lange, sich von den umgebenden mononucleären Zellen abhebende Züge bilden.

Die zwischen dem infiltrirten Gewebe eingeschlossen liegenden Skleralparthieen, welche noch nicht von der Entzündung ergriffen sind, scheinen auch nicht mehr ganz normal zu sein, sondern zeigen hier und da eine geringe oedematöse Quellung und Auflockerung.

Auf der andern, äusseren Hälfte ist das Bild insofern etwas anders, als zunächst die Vermehrung und Ausbreitung der ektatischen Lymphgefässe hier einen viel bedeutenderen Grad erreicht hat und vor der Blutgefässentwicklung entschieden prävalirt, wenn auch die Ektasie der einzelnen Lymphgefüsse an sich nicht so auffallend als auf der andern seite ist. Die Lymphgefässbildung ist hier in so ausgedehnter Weise erfolgt, dass gewisse Stellen (Fig. 2 u. 4, k) völlig das Aussehen cavernösen Gewebes erhalten haben. Die Ausdehnung der so veränderten Subconjunctiva beträgt von vorn nach hinten ca. $3 \mathrm{~mm}$. Ferner ist in der Sklera selbst die Infiltration im Allgemeinen viel diffuser. Es bestehen zahlreiche kleinere theils streifen-, theils heerdförmige Infiltrate, welche in mehr gleichmässiger Weise sämmtliche Sklerasehichten durchsetzen. Aus der Suprachorioidea stammendes Pigment ist hier bis mitten in die Sklera versehleppt, so dass eine scharfe Abgrenzung der Sklera von der Suprachorioidea ganz unmöglich ist. An mehreren Stellen sind jedoch auch in dieser Hälfte normale Theile der Sklera erhalten geblieben. So hat sich unmittelbar an der Corneoskleralgrenze ein grösserer dichter Infiltrationsheerd gerade in der Mitte der Sklera etablirt, der die normalen Sklerafasern bogenförmig nach vorn und hinten auseinanderdrängt. Die vorderen Sklerabündel verlaufen continuirlich von vorn nach hinten, die hinteren sind an zwei Stellen unterbrochen und hören, sich zerfasernd, ziemlich plötzlich auf. Vor beiden Stellen liegen wieder kleine nekrotische Parthieen mit theils typisehen, theils atypischen Riesenzellen in ihrer Umgebung. Und ebenso wird mehr nach hinten zn die diffuse Infiltration unterbrochen, indem ganz unvermittelt mitten in derselben ein Stück anseheinend ganz normaler Sklera auftritt, das 
vorn sich scharf, wie abgeschnitten, gegen das infiltrirte Gewebe absetzt, nach hinten dagegen bald seine normale Beschaffenheit verliert und von zahlreichen Leukocyten durchsetzt wird. Oberund unterhalb dieses Stückes normaler Sklera sind die Infiltrate wieder dichter und fliessen, ähnlich wie auf der anderen Seite, zu compacten Massen zusammen, welche auch hier sich nach hinten zu bis zum Ansatz der Muskelsehne fortsetzen. In der tiefen nach innen von der Sklera gelegenen Infiltration befinden sich ganz besonders zahlreiche und ansgedehnte, zum Theil miteinander communicirende nekrotische Parthieen. Verfolgt man dieselben in den einzelnen Schnittserien weiter, so findet man, dass auch diese Nekrosen einen vollständigen Ring um den ganzen Bulbus bilden, indem die einzelnen Heerde plexiform miteinander zusammenhängen. Am wenigsten intensiv ist diese Gewebsnekrose in der oberen Bulbushälfte ausgebildet, indem in ihren mittleren Parthieen nicht eigentliche Heerde mehr $\mathrm{za}$ finden sind, sondern sich nur die den Kreis schliessenden Auslänfer benachbarter grösserer Heerde hinziehen.

Es erübrigt noch, diejenigen Theile des Bulbus zu betrachten, welche in keiner so nahen Beziehrung zu der geschilderten Infiltrationszone des Limbus stehen. Die in die vordere Kammer luxirte Linse weist, wenn man von jenen artificiellen Defecten an der Vorderläche absieht, überall normalen Kapseluiberzag auf. Hier und da findet sich unter der Kapsel etwas Morgagnische Flüssigkeit angesammelt. Am Linsenrande sieht man zahlreiche Vacuolen die Linsenfasern durchsetzen. Der etwas dunkler gefärbte Kern bietet nichts Besonderes.

Die nach hinten zurückgeschlagene Iris ist, wie schon erwähnt, der Sitz einer mässigen diffusen Infiltration, welche jedoch an einzelnen Stellen eine haufenweise Anordnung zu circumseripten Infiltrationsheerden erkennen lässt (Fig. 4, b). Die Iris ist mässig sklerosirt und zeigt eine starke Atrophie, so dass sie trotz der zelligen Infiltration durchschnittlich etwa anf die Hälfte des normalen Volumens reducirt erscheint, stellenweise noch erheblich mehr.

Auch die intensive zellige Infiltration des Ciliarkörpers ist schon besprochen worden. Hinter demselben hört die Infiltration der Chorioidea sehr bald anf, und es finden sieh in den hinteren Theilen der Chorioidea nur noch einzeln verstrente Lenkoeyten, die nur an wenigen Stellen sich in Gruppen zu kleinen Infiltrationsheerden vereinigen. Dagegen hat in der ganzen Ausdehnung der Chorioidea eine reichliche ödematöse Durch- 
tränkung Platz gegriffen, welche dieselbe in gewissen Parthieen anf das mehrfache ihres ursprünglichen Volumens verbreitert hat, so z. B. unmittelbar hinter dem Corpus ciliare. Hier hat sich zwischen den Lamellen der Chorioidea und Suprachorioidea eine mächtige Transsudatmasse etablirt, welehe die Hauptmenge der Faserbündel jener Membranen von der Sklera abdrängt und nur die Lamina fusca in Verbindung mit ihr gelassen hat. Die abgehobenen Theile sind anch ihrerseits wieder durch geronnene Oedemflüssigkeit in einzelne Gewebslamellen zerklüftet, bisweilen so stark, dass die aufgefaserten und auseinandergedrängten Chorioidealschichten auf dem Schnitte wie ein aus Fäden gebildetes Netzwerk erseheinen (Fig. 4, $m$ ). Die Gefässe sind, besonders nach hinten $z u$, strotzend gefüllt.

Was endlich die Netzhaut und den Sehnerven betrifit, so ist letzterer fast vollständig atrophisch. Die Nervenfasern haben durchweg ibr Mark verloren, und nur an wenigen Stellen lassen sich noch Nervenelemente nachweisen; zum grössten Theil ist nur noch das zarte Glianetz übrig geblieben. Die Papille selbst ist in ihrer äusseren Gestalt ziemlich normal. In ihrer Nähe sind die Nervenfasern der Netzhaut noch zieralich gut erhalten, obgleich man auch da die Gliafasern stellenweise deutlich erkennen kann. Mehr nach vorn zu lassen sich dann gar keine Nervenfasern mehr nachweisen. Die Membrana limitans interna ist stark verdiekt, die Stäbchen und Zapfen bisweilen in radiären Büscheln angeordnet. Im Uebrigen zeigt die Netzhaut ödematöse Veränderungen und zwar zunächst hinten in unmittelbarer Nähe des Sehnervenkopfes. Hier ist der Sitz der intensivsten Veränderungen die äussere reticuläre Schicht, welche die bekannten eharakteristischen Lüeken aufweist mit dem zu Bündeln angeordneten ausgezogenen Stützgewebe dazwisehen. Doch auch die ibbrigen Netzhautsehichten sind mehr oder weniger stark in Mitleidenschaft gezogen. Bemerkenswerth ist, dass auch die durch eine kleinere Exsudation abgehobene Macula lutea von dieser cystoiden Entartung mit betroffen ist. Eine ähnliche Cystenbildung, wie in den hinteren Parthieen, hat sich auch in der Pars ciliaris retinae etablirt und greift aueh auf die spornartig vorspringende Ora serrata über (Fig. $4, n$ ); sie nimmt von der Iriswurzel nach der Ora serrata hin, also von vorn nach hinten, an Intensität zu. In den vorderen, weniger hochgradig veränderten Parthieen ist noch die pallisadenförmige Anordnung der Epithelzellen im Grossen und Ganzen wohl erhalten, obgleich sich anch hier sehon an zahlreichen Stellen schmale Lücken. 
zwischen die einzelnen Zellen schieben und sie auseinander drängen. Dagegen ist der regelmässige Bau der an die Ora serrata grenzenden Theile völlig aufgehoben. Hier ist das Stützgewebe zu feinen Fäden ausgezogen, welche die Scheidewände von grossen unregelmässig gestalteten Hohlränmen, die sich hier gebildet haben, darstellen. Zum Theil sind diese schmalen Scheidewände zwischen den Cystenlumina sogar in ihrer Continuität unterbrochen, so dass ilhre freien Enden in die Hohlräume ragen. Die Kerne der Epithelzellen finden sich alle in den basalen Schichten, in den cystoiden Parthieen liegen nur vereinzelte Leukocyten, welche augenscheinlich aus dem benachbarten Glaskörper dorthin eingewandert sind.

In der äusseren Hälfte hat sich dicht hinter dem Corpus ciliare zwischen den abgehobenen Schichten der Retina einerseits und dem mit der Chorioidea fest verlöteten Pigmentepithel andererseits eine gefässführende entzündliche Bindegewebsschichte gebildet, welche sich nach hinten verjüngt (Fig. 4, o). Die Netzhaut ist von ihr durch Hohlräume getrennt and hängt nur stellenweise durch schmale Brücken mit ihr zusammen, so dass dadureh die bekannten arkadenartigen flachen Bogen entstehen. Die Schwarte selbst besteht aus faserigem, von zahlreichen Rundzellen durchsetztem Bindegewebe und ist zum Theil auch durch Transsudatmassen von der Chorioidea abgehoben. Die in ihr bestehende Infiltration setzt sich nach vorn zu fort und geht dort continuirlich in die infiltrirte Chorioidea über.

In dem Glaskörper, soweit derselbe erhalten ist, finden sich spärliche mono- und polynucleäre Leukocyten.

Resumiren wir kurz die wichtigsten der gefundenen pathologischen Veränderungen unter Berücksichtigung dex bereits von anderm Autoren bei verwandten Erkrankungen beschriebenen Befunde, so haben wir zunächst in der Hornhaut einen Pannus, wie er ähnlich von Baumgarten ${ }^{\mathbf{1}}$ ) in einem Falle sklerosirender Keratitis beobachtet wurde. Wir haben ferner, ebenfalls in der Hornhaut, jedoch nur mehr nach der Peripherie hin, zahlreiche kleinere Infil-

1) Baumgarten, Ophthalmolog. - histologische Mittheilungen. I. Ein Fall von sklerosirender Keratitis. v. Graefe's Archiv f. Ophthalm. 1876. Bd. XXII. 2. S. 185-203. 
trate, als Ausläufer der grösseren, massigen Zellanhäufungen in und hinter der Corneoskleralgrenze. Und zwar sind sie hier vorzugsweise streifenförmig angeordnet, in langen perlschnurartigen Reihen die Interstitien der Hornhautlamellen erfüllend, ein Bild, für das Grayet ${ }^{1}$ ) einen sehr hübschen und treffenden Vergleich findet, indem ex sagt: $"$... il résulte de leurs (des lymphoides) dispositions une sorte d'élégante chinure". Und wir haben endlich ein verdicktes Epithel, das sich zapfenförmig in die Tiefe des Hornhantparenchyms senkt. Letzterer Befund ist wieder dem bei Gayet ${ }^{2}$ ) angegebenen analog: ". . . l'epithélium bouleversé enfonce dans les couches profondes de gros prolongements papilliformes". Jedoch erwähnt der genannte Autor nicht, ob dort auch eine reactive Entzïndung des die Zapfen umgebenden Hornhautgewebes bestanden habe. Die vermehrte Bildung ektatischer Lymph- und Blutgefässe scheint nach den bisherigen Beobachtungen bei Skleritis und Episkleritis eine constante Erscheinung zu sein; und zwar betont schon Uhthoff'), was auch hier der Fall ist, dass die Lympfgefässe stets ,in den oberflächlichsten Conjunctivalschichten ziemlich nahe unter dem Epithelium" liegen. Hier scheint jedoch diese Vermehrung, zumal die der Lymphgefässe, eine ganz besonders starke zu sein; täuscht sie doch auf eine ziemlich ausgedehnte Strecke hin das Bild cavernösen Gewebes vor! Und hierin haben wir augenscheinlich die anatomische Ursache für jenes so charakteristische klinische Bild der sulzigen, succulenten Verdickung zu suchen, das uns bei allen drei Fällen mit bemerkenswerther Uebereinstimmung begegnet ist.

Als die markanteste und eingreifendste Veränderung,

1) L. c. S. 23 .

2) L. c. ibidem.

3) Uhthoff, Beiträge zur pathologischen Anatomie des Auges. I. Skleritis und Episkleritis. v. Graefe's Archiv f. Ophthalm. 1883. Bd. XXIX. 3, S. 167-173. 
welche auch ron den bisher beschriebenen ähnlichen pathologischen Zuständen ganz abweichend ist, stellt sich jene colossale leukocytäre Infiltration dar, welche ringförmig den ganzen Limbus einnimmt und in sich die gleichfalls einen geschlossenen Ring bildenden plexiform zusammenhängenden nekrotischen Heerde schliesst. Diese Infiltration scheint die Sklera an vielen Stellen ziemlich jäh durchbrochen zu haben, da die Sklerafasern oft plötzlich und unvermittelt aufhören und sich scharf gegen die infiltrirte Umgebung abgrenzen. Ebenfalls merkwürdig und höchst auflallend ist der enorme Reichthum an Riesenzellen, der in diesem Grade bisher auch noch nicht beobachtet zu sein scheint.

Die übrigen pathologischen Veränderungen sind zweifellos secundärer Natur und daher für das anatomische Krankheitsbild von untergeordneter Bedeutung. Der primäre Sitz der Entzündung im Augeninnern dürfte die Chorioidea sein. Von ihr hat der Process auf das Corpus ciliare und die Tris übergegriffen und $z u$ einer Seclusio pupillae mit sich daranschliessendem Secundärglaukom geführt. Ferner ist es durch starke Exsudation zu einer Abhebung der Retina und Chorioidea gekommen. Die Netzhaut, zum Theil auch ihrerseits wieder von der Chorioidea abgehoben, ist einer vollständigen Degeneration anheim gefallen, die ascendirend auch den Sehnerven in Mitleidenschaft gezogen und seine fast totale Atrophie veranlasst hat.

Bei der Frage nach dem Wesen der geschilderten Krankheit, nach den ätiologischen Momenten, die dabei im Spiele sein mögen, kommen wir einigermaassen in Verlegenheit, denn es lässt sich nicht leugnen, dass die Aetiologie recht dunkel ist. Mir scheint die vorliegende Erkrankungsform gewisse unverkennbare Beziehungen zu der sog. tiefen Skleritis zu haben. Ich rechne dazu u. a. die diffuse Ausbreitung des Processes, sowie die Betheiligung auch der tiefen Skleralschichten an demselben; und auch einige andere 
Punkte, besonders gewisse anatomische Verhältnisse erinnern entschieden an Skleritis. Gegen dieselbe spricht jedoch, wie schon oben des Weiteren ausgeführt ist, die zum mindesten ungewöhnliche Localisation und Ausbreitung der Entzündung. Auch ist es auffallend, dass alle drei Fälle alte Männer betreffen, während die tiefen Formen der Skleritis doch rorwiegend jüngere Individuen weiblichen Geschlechtes zu befallen pflegen.

Die rein anatomische Untersuchung hat für die Entscheidung der Frage nach der Aetiologie zunächst nur negative Resultate ergeben. Tuberculose kann mit ziemlicher Sicherheit ausgeschlossen werden, denn weder liess irgend eine Stelle den typischen Bau des Tuberkelknotens erkennen, noch wurden Tuberkelbacillen gefunden; ausserdem bot auch die interne Untersuchung keinerlei Anhaltspunkte für diese Annahme. Gleichfalls absehen kann man von Syphilis, da weder in den anamnestischen Angaben, noch bei der objectiven Untersuchung, noch auch endlich in den histologischen Verhältnissen irgend etwas darauf hindeutet; die drei Fälle von Gunn lehren überdies, dass das klinische Bild bei syphilitischer Infiltration des Limbus doch in mancher Hinsicht von dem unsrigen abweicht. Von Tumoren konnte nirgends etwas constatirt werden, und ebenso wenig handelt es sich offenbar um Pseudoleukämie oder verwandte constitutionelle Erkrankungen, einmal wegen der negativen Resultate, zu welchen die Untersuchungen des Blutes intra vitam und in den Schnitten geführt haben, und ferner auch, weil es kaum denkbar ist, dass eine derartige Krankheit mit der Vernichtung eines Auges ihren Abschluss gefunden hätte. Allenfalls verdient die bei zweien der Fälle gemachte anamnestische Angabe Berücksichtigung, dass Rheumatismus bestanden habe. Doch wird man auf diese so häufig wiederkehrende Angabe allzu grosses Gewicht nicht legen dürfen.

Giebt die anatomische Untersuchung demnach auch 
keine directe positive Antwort auf die Frage nach den möglicherweise in Betracht kommenden ätiologischen Momenten, so bieten doch gewisse histologische Verhältnisse einen Ausgangspunkt für Betrachtungen über die eventuellen Endursachen des Krankheitsprocesses. Und zwar möchte ich an die entschieden auffallende Menge von Riesenzellen anknüpfen, welche überall da zu finden waren, wo die Entzündung einen höheren Grad erreicht hatte, d. h. in der Chorioidea, Suprachorioidea, Sklera, Episklera und - ein nicht ganz gewöhnlicher Befund - auch in der Cornea. Besonders constant fanden sich dieselben in der Peripherie jener nekrotischen Parthieen, and man könnte sie vielleicht als eine Folge dieser Gewebsnekrosen auffassen, wie sich ja häufig an die Oberfläche solcher nekrotischer Heerde Riesenzellen anlagern, als ein Ausdruck vermehrter Kerntheilung bei behinderter Protoplasmatheilung. Dagegen spricht jedoch der Umstand, dass die Riesenzellen sich auch an anderen Stellen vorfanden, in Geweben, wo es zu einer Nekrose überhaupt nicht gekommen ist (Episklera, Cornea!). Falls man daher nicht zu der etwas gezwungenen Annahme greifen will, dass die verschieden localisirten Riesenzellen auch verschiedenen Ursachen ihre Entstehung verdanken, so wird man zu dem Schluss kommen, dass Nekrose und Bildung von Riesenzellen die Folge des nämlichen Processes sein müssen, dass sie als gleichwerthige Ausdrücke eines und desselben ursächlichen Momentes aufzufassen sind. Dabei kann es sich aber nur um Bakterien handeln. Diese können einmal als einfache, einen mechanischen Reiz setzende Fremdkörper wirken - und erregen dann Entzüudung und Gewebswucherung, und es kommt in ihrer unmittelbaren Umgebung zur Bildung von Riesenzellen; ferner aber wirken sie auch durch ihre chemischen Umsetzungsproducte auf das ihnen benachbarte Gewebe ein - und führen dann zu Nekrose und Degeneration. 
Mir ist es demnach wahrscheinlich, dass wir es hier mit einer mykotischen Frkrankung zu thun haben, mit einer Entzündung, hervorgerufen durch die Gegenwart irgendwelcher Mikroorganismen. Welcher Art dieselben sein mögen, darüber lässt sich freilich bei dem Versagen der gebräuchlichen bakteriologischen Färbungsmethoden nichts Sicheres angeben, und wir müssen uns daher vor der Hand damit begnügen, von späteren Untersuchungen eine weitere Aufklärung in dieser Richtung zu erhoffen.

Herrn Prof. Fuchs sage ich auch an dieser Stelle noch meinen aufrichtigsten Dank für seine liebenswürdige, jederzeit bereite Unterstützung bei meiner Arbeit.

\section{Erklärung der Abbildungen auf Tafel III.}

Fig. 1. Uebersichtsbild über den Bulbusdurchschnitt (nach einem Schnitte gezeichnet).

a In die vordere Kammer luxirte Linse.

$b$ Nach hinten zurückgedrängte Iris.

c Zone der Infiltration in Sklera, Episklera, Suprachorioidea und Corpus ciliare.

$d$ u. $e$ Durch Transsudatmassen von der Sklera abgehobene Chorioidea und Retina.

$f$ Atrophischer Sehnerv.

Fig. 2. Vermehrte und ektatische Lymphgefässe, in ihrem Aussehen an cavernöses Gewebe erinnernd (cf. auch Fig. 4, $k$ ).

Fig. 3. Nekrotischer Heerd mit Riesenzellen an seiner Peripherie (cf. auch Fig. 4, i).

Fig. 4. Topographische Ansicht des vorderen Bulbusabschnittes. (Die Linse hatte sich beim Durchschneiden des Augapfels etwas verschoben, so dass die durch die Trepanation hervorgerufenen Defecte in der Linse nicht in allen Schnitten dem Hornhautdefect entsprechen):

a Epithelzapfen im Hornhautparenchym.

$b$ Infiltration in der Cornea, die Bowman'sche Membran durchbrechend.

c Ringförmiges Infiltrat in den hinteren Hornhautlamellen, mit der obertächlichen Inflttration längs der Gefässe communicirend. 
82 W. Schlodtmann, Ueber sulzige Infiltration d. Conjunetiva u. Sklera.

d Oberflächliches Infiltrat in der Subconjunctiva, Episklera und den äusseren Skleralschichten.

$e$ Tiefes Infiltrat in Corpus ciliare, Suprachorioidea und inneren Skleralschichten.

$f$ Starke Ektasie der Lymphgefässe.

$g$ Normale Sklera.

$h$ Verbindender Infiltrationsstreifen zwischen oberflächlichem und tiefem Infiltrat, längs eines Gefässes hinziehend.

$i$ Nekrotische Stellen.

to Vermehrte Lymphgefässbildung, von dem Aussehen cavernösen Gewebes.

$l$ Circumseriptes Infiltrat in der Iris.

$m$ Durch Oedemflüssigkeit lamellirte Chorioidea.

$n$ Cystoide Entartung der Pars ciliaris retinae.

- Cyclitische Schwarte unter der Netzhaut. 
S

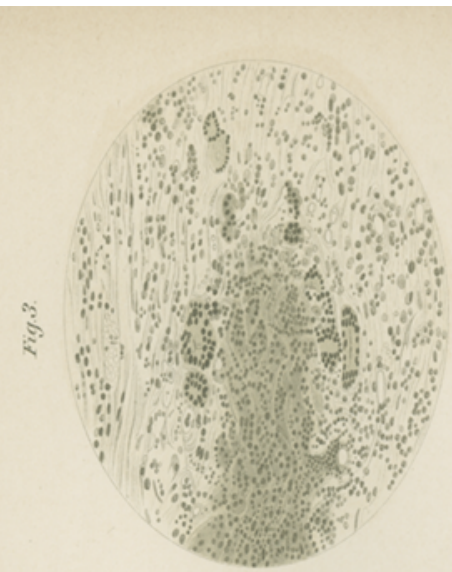

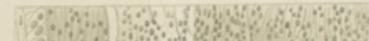

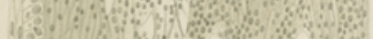
S.N. 0 \% 3.

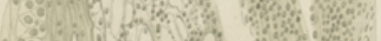

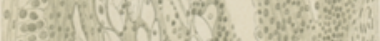

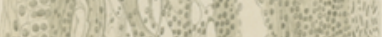

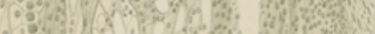

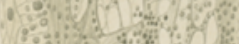

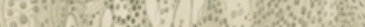

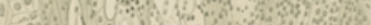

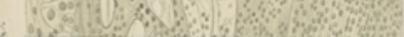

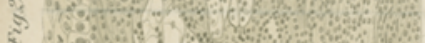

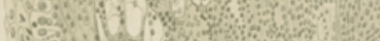

190.6.

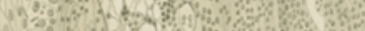
150:30\%:

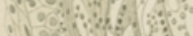

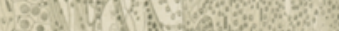

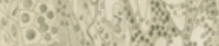

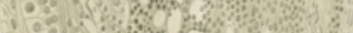

21.6.5.

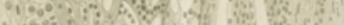

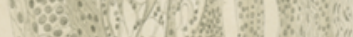

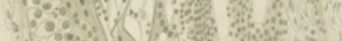

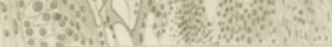

है 